\title{
RESENHA DO LIVRO: "LONG-RUN ECONOMIC RELATIONSHIPS: READINGS IN COINTEGRATION" DE ROBERT F. ENGLE E CLIVE W. J. GRANGER*
}

\author{
João Victor Issler**
}

\section{Motivação.}

O objetivo desta seção é introduzir o conceito de co-integração de uma forma intuitiva para os leitores não-familiarizados com os mais recentes avanços na literatura em séries temporais. Busca-se tambćm integrar este conceito com várias questões relevantes em análise de regressão em séries temporais. O leitor familiarizado com esse material pode iniciar a leitura na seção seguinte.

Um dos problemas óbvios a qualquer um que tenha realizado trabalhos empíricos com séries temporais é o seguinte: enquanto as hipóteses básicas da análise de regressão exigem séries estacionárias e ergódicas para obter-se estimadores e estatísticas bem comportados, as séries disponíveis ao pesquisador normalmente têm os dois primeiros momentos não-estacionários. Como conseqüência, a aplicação da análise de regressão a séries desse tipo pode potencialmente levar a resultados desastrosos. Por exemplo, o trabalho de Granger e Newbold (1974) em regressões espúrias mostra que a estatística $t$, obtida em regressões envolvendo. duas séries de passeios aleatórios independentes, é em média demasiadamente alta relativamente a valores críticos usuais. Logo, seu uso levaria muito freqüentemente a conclusão de que duas séries independentes são correlacionadas.

O trabalho de Nelson e Plosser (1982) influenciou uma gcração de macroeconometristas a modclarem a não-estacionaridade das séries temporais como tendências estocásticas, i.e., séries macro seriam processos integrados. Se denotarmos processos "estacionários" (nãointegrados) como $\mathrm{I}(0)$, define-se $\left\{y_{t}\right\}$ como um processo integrado

\footnotetext{
*Engle, Robert F. \& Granger, C. W. J. editores, 1991. Long-Run Economic Relationship: Readings in Cointegration. Oxford: Oxford University Press, 301 pp. ISBN: 0-19-828338-5.

**Department of Economics, University of California at San Diego.
}

R. de Econometria Rio de Janeiro v. 12, no 2 , pp.241-248 novembro 1992 
de ordem $k[I(k)]$ se $\Delta^{k} y_{t}$ for $I(0)$. A literatura atual se concentra no caso de processos $I(1)$, porém também se estuda o caso $I(2)$. $O$ processo $I(1)$ mais simples é o passeio aleatório:

$$
y_{t}=y_{t-1}+\varepsilon_{t}, \quad \text { onde } \varepsilon_{t} \sim i \cdot i \cdot d \cdot\left(0, \sigma^{2}\right) .
$$

Nesse caso, $\Delta y_{t}=\varepsilon_{t}$ é um processo $I(0)$. Note também que podemos escrever $y_{t}=\Delta^{-1} \varepsilon_{t}=\sum_{i=0}^{t-1} \varepsilon_{t-i}$, caso $y_{0}=0 ;$ o que mostra que $\left\{y_{t}\right\}$ contém uma tendência (integral) aleatória $\sum_{i=0}^{t-1} \varepsilon_{t-1}$ ). Como esta tendência é uma acumulação de todos os choques passados com pesos idênticos, a série $\left\{y_{t}\right\}$ tem memória longa e aparência regular. Ademais, sua variância é igual a $t \cdot \sigma^{2}$ aumentando sem limite com $t$.

Para duas séries de igual ordem de integração, e.g. I(1), o conceito de co-integração diz respeito à existência de uma combinação linear dessas séries que tem a propriedade de reduzir essa ordem, e.g. ser $I(0)$. Neste caso, a tendência estocástica presente em cada série isoladamente é eliminada pela combinação linear, logo era comum às duas séries. $\mathrm{O}$ conceito de co-integração tem estreita ligação com o equilíbrio de longo prazo em relações econômicas. Por excmplo: a teoria da renda permanente de Friedman diz que indivíduos consomem uma proporção de sua renda permanente e não de sua renda corrente. Empiricamente, testes de integração em renda e consumo percapita indicam que essas variáveis são $I(1)$, logo ambas contêm tendências estocásticas. Se denotarmos a tendência estocástica da renda como "renda permanente," uma condição necessária para a teoria de Friedman ser atendida é que consumo e renda co-integrem, isto é, que desconsiderados componentes transitórios em ambos, a parte permanente do consumo seja proporcional à renda permanente. Caso essas duas variáveis sejam idênticas, o vetor de co-integração é $(1,1)$.

A sugestão de Granger e Newbold (1974) para trabalhar com séries integradas era a de tomar as primeiras diferenças dos dados antes de utilizar a análise de regressão. Satisfazia-se assim o requisito de "estacionaridade" $[I(0)]$. Esta sugestão foi seguida por vários autores, inclusive Christopher Sims, que encabeça a literatura de vetores Auto-regressivos (VAR's). Embora usar dados em diferenças 
resolva o problema de "estacionaridade" dos dados das regressões, pode-se perder informações relevantes sobre relações de longo prazo entre variáveis econômicas. A seguinte análise ilustra este problema. Suponha que o processo gerador de $\left\{y_{t}\right\}$ e $\left\{z_{t}\right\}$ seja o seguinte:

$$
\begin{aligned}
& Y_{t}=\beta_{1} z_{t}+\beta_{2} z_{t-1}+\beta_{3} y_{t-1}+\varepsilon_{1 t} \\
& Z_{t}=Z_{t-1}+\varepsilon_{2 t}
\end{aligned}
$$

onde $\left|\beta_{3}\right|<1, \quad \varepsilon_{1 t} \sim \operatorname{IIN}\left(0, \sigma_{2}^{2}\right), \quad \varepsilon_{2 t} \sim \operatorname{IIN}\left(0, \sigma_{2}^{2}\right)$, $\operatorname{COV}\left(\varepsilon_{1 t}, \varepsilon_{2 t}\right)=0$

Aqui, $\{z$,$\} é I(1), dado que é um passeio aleatório. \left\{y_{t}\right\}$ também o é, dado que o processo gerador de $\left\{y_{t}\right\}$ contém $\left\{z_{t}\right\}$. Existe clara. mente co-integração entre $\left\{y_{t}\right\}$ e $\left\{z_{t}\right\}$, dado que estas variáveis estão ligadas pela seguinte relação de longo prazo:

$$
y=K \cdot z, \quad \text { onde } \quad K=\frac{\beta_{1}+\beta_{2}}{1-\beta_{3}}
$$

Transforme agora (1) da seguinte forma:

$$
\begin{aligned}
\Delta y_{t} & =\beta_{1} Z_{t}+\beta_{2} z_{t 1}+\left(\beta_{3}-1\right) y_{t_{1}}+\varepsilon_{1 t}, \\
& =\beta_{1} \Delta z_{t}+\left(\beta_{1}+\beta_{2}\right) z_{t-1}+\left(\beta_{3}-1\right) y_{t-1}+\varepsilon_{1 t} \\
& =\beta_{1} \Delta z_{t}+\left(\beta_{3}-1\right)(y-K \cdot z)_{t-1}+\varepsilon_{1 t}
\end{aligned}
$$

Podemos agora discutir a sugestão de Granger e Newbold: estimando-se uma regressão de $\Delta y_{t}$ em $\Delta z_{t}$, omite-se somente a relação de longo prazo $(y-K \cdot z)_{t-1}$, o que causa problemas do tipo variáveis omitidas em regressões (potencialmente presentes também em VAR's em 1르 diferença). Estimando-se porém o Modelo de Correção de Erros (MCE) (4) utilizam-se todas as informações contidas em (1), com a vantagem que todas as séries em (3) são processos "estacionários", livres portanto dos problemas anteriormente mencionados. Como (4) é apenas uma reparametrização de (1), esta situação é um caso especial de variáveis integradas, onde estas co-integram gerando estimadores e estatísticas bem comportadas. No entanto, se estimarmos o MCE ao invés de (1) há um ganho de eficiência, dado que nele, estamos impondo, a relação de longo prazo entre $y$ e $z$. 


\section{Resenha de Engle e Granger.}

O livro é a mais completa coletânea de artigos sobre co-integração existente no momento, conceito usado hoje na grande maioria dos trabalhos empíricos que utilizam métodos econométricos em sćries temporais. O conceito de co-integração foi introduzido por Clive W. J. Granger em 1981. A maioria dos artigos da coletânea foi publicada nos últimos seis anos (ver lista ao final da resenha). Além de artigos publicados, o livro traz quatro artigos não publicados, Granger [1,14], Engle e Yoo [12] e MacKinnon [13], todos inéditos exceto o último.

O livro pode scr utilizado por uma ampla audiĉncia que vai de pesquisadores na fronteira dos assuntos em sćries temporais a pcsquisadores que tenham algum interesse em trabalhos empíricos mas não estão atualizados a respeito dos avanços em séries temporais. Uma sólida noção básica de econometria é necessária para a leitura do texto.

Há três artigos introdutórios sobre co-integração e suas implicações: a introdução de Granger, Stock and Watson [2] e Hendry [3]. Estes trabalhos são bastante abrangentes e dão uma boa noção da importância de se tomar cuidados especiais com séries macroeconômicas integradas c co-integradas. Tanto Hendry [3], como Stock e Watson [2] discutem o problcma de se usar variáveis integradas em "nível" em regressõcs lincares. É particularmente interessante 0 experimento "Monte Carlo" usado pelos últimos para ilustrar esses problemas. A ligação entre teoria econômica e co-integração é discutida por Granger na introdução, usando o conceito de atração entre variáveis econômicas.

$\mathrm{O}$ artigo intermediário do livro é Granger [4]. A meu ver, esse é um dos artigos mais bem escritos do livro. É o artigo intermediário perfeito: define os conceitos fundamentais de forma intuitiva $€$ discute as implicações de forma profunda. Contém um dos resultados mais importantes de co-integração (Teorema de Representação de Granger): a saber que, qualquer Vetor Auto-regressivo (VAR) em níveis contendo variáveis $I(1)$ pode ser represcntado como um Modelo de Correção de Erros (MCE). Portanto, para cste tipo de VAR, tomar as primeiras diferenças dos dados e depois cstimar o VAR, omite informações de longo prazo contidas nos termos de correção de erros. 
Outros pontos importantes deste trabalho são: relação entre causalidade de Granger e co-integração, implicações de co-integração para projeções e a introdução do conceito de multi-co-integração, discutido em detalhe em Granger e Lee [9]. O exemplo clássico de multico-integração utiliza as séries de vendas e produção para uma empresa. Normalmente a variação de estoques de uma firma (vendas menos produção) é um processo $I(0)$, logo vendas e produção cointegram com vetor $(1,1)$. Como a variação de estoques é $I(0)$, o nível de estoques é $I(1)$. Quando vendas, produção e nível de estoques co-integram diz-se que existe multi-co-integração entre vendas e produção.

$\mathrm{O}$ artigo seguinte de Engle e Granger [5], é fundamental para a literatura de co-integração: primeiro, prova-se formalmente o Teorema de Representação de Granger. Segundo, discute-se um teste de co-integração em dois estágios muito simples, baseado em estimação via OLS e usando a estatística de Dickey e Fuller (1979). Este teste de dois estágios é extensivamentc usado na literatura macroeconômica atual. Uma das implicações do resultado do teste de co-integração proposto por Engle e Granger é que se pode substituir termos de correção de erros em MCE's por suas séries estimadas, via o teste de dois estágios, dado que o vetor de co-integração estimado é supcrconsistente, convergindo para o paramêtro populacional a uma taxa mais rápida que o usual.

O trabalho de Engle e Yoo [6] discute projeções de um sistcma VAR I(1) e co-integrado. Usando "Monte Carlo," compara-se o Erro Quadrático Médio (EQM) de um sistema usando um MCE com a restrição de longo prazo do teste Engle-Granger com o obtido via a estimação do VAR de forma irrestrita. Conclui-se que a longo prazo o EQM do primeiro é menor, dado que se impõe no VAR as restrições advindas da co-integração. $O$ trabalho também apresenta valores críticos para o teste de co-integração. Valores mais precisos são encontrados em MacKinnon [13].

O artigo de Johansen [7] é um dos mais importantes para o completo entendimento do fenômeno de co-integração. Baseado em um sistema multivariado $I(1)$ com representação de VAR e erros normais, o autor propõe um teste para o posto de co-integração e uma 
forma de estimar uma base para o subespaço gerado pelos vetores de co-integração. Estimações são feitas pelo método de máxima verossimilhança sob normalidade. Todavia, como as variáveis que integram o VAR são $I(1)$, a distribuição assintótica do teste de posto é não-padrão. Mais explicitamente, é uma função de integrais de Movimentos.Brownianos, dado que a parte integrada de variáveis $I$ (1) converge em distribuição para esse tipo de integral. Boa parte do texto é dedicada às propriedades assintóticas do teste de posto e dos estimadores do subespaço de co-integração. Para entender esta parte, é necessário um bom conhecimento de teoria assintótica e estatística multivariada. A importância do método de Johansen é que este possibilita estimar o posto e o subespaço de co-integração, o que tem enorme utilidade para sistemas multivariados com mais de duas variáveis. $\mathrm{O}$ método também possibilita discutir-se co-integração de sistemas contendo conjuntamente variáveis $I(2), I(1)$ e $I(0),{ }^{1}$ o que é muito útil para sistemas com variáveis macroeconômicas nominais: moeda, preços, taxas de câmbio, etc.

O trabalho de Stock e Watson [8] tem relevância teórica por provar que um sistema multivariado de $N$ variáveis $I(1)$ com posto de co-integração $r<N$, contém uma representação com $N-r$ tendências comuns, definidas como um passeio aleatório com drift. Este mesmo resultado é apresentado em Engle e Yoo [6] de forma bem mais intuitiva. Stock e Watson também propõem um teste de co-integração baseado em OLS, porém que não é tão utilizado em trabalhos empíricos quanto os testes de Engle-Granger e Johansen.

$\mathrm{O}$ artigo de Engle e Yoo [12] usa a forma Smith-McMillan de decomposição de matrizes polinomiais para diṣcutir diferentes representações de sistemas multivariados de variáveis $I(1)$ (o caso de $I(2)$ é também discutido). Discute-se o caso da representação de vetor MA, vetor $A R$ e co-integracão polinomial, onde os termos de correção de erros entram com defasagens múltiplas. Um método de estimação de co-integração em três estágios é também proposto.

Os artigos de Campbell e Shiller [10] e Engle, Granger e Hallman [11] não têm a mesma importância dos listados acima. Talvez o primeiro se sobressaia nessa lista, dado que interpreta co-integração

$\overline{1}$ Ou em geral de ordens de integração 
usando o conceito de valor presente. Mesmo assim, sua leitura não empolgará o leitor que ler o livro inteiro. O artigo final de Granger [14] apresenta tópicos interessantes representando extensões de co-integração. Sobre alguns destes tópicos, como por exemplo cointegração sazonal, já existe algo escrito a respeito. Porém outros tópicos, como agregação e co-integração não-linear, podem ainda se tornar mais difundidos na literatura sobre co-integração.

Penso que esse livro é leitura obrigatória para qualquer econometrista interessado em séries temporais. Ademais, como a macroeconomia atual utiliza cada vez mais métodos de sérics temporais modernos, macroeconomistas aplicados se beneficiarão muito de sua leitura. Houve um tempo em que os métodos econométricos eram completamente desvinculados de teoria econômica e de uma metodologia de pesquisa globalmente aceita. A literatura de co-integração se constituiu num passo significativo em busca do estreitamento da ligação entre teoria econômica e estimação econométrica, tornando a primeira mais testável e os resultados da segunda mais criveis.

\section{Lista de artigos (capítulos) do livro.}

[1] Clive W. J. Granger Introduction.

[2] Stock, J. \& Watson, M. 1988. "Variable trends in economic time eeries." Journal of Economic Perspectives 2:147-174.

[3] Hendry, D.F. 1986. "Economic modelling with cointegrated variables: an overview." Oxford Bulletin of Economics and Statistics 48:201-212.

[4] Granger, C.W.J. 1986. "Developments in the study of cointegrated economic variables." Oxford Bulletin of Economics and Statistics 48: 213-228.

[5] Engle, R.F. \& Granger, C.W.J. 1987. "Co-integrated and error correction: representation, estimation and testing." Econometrica 55: 251-276.

[6] Engle, R.F. \& Yoo, B.S. 1987. "Forecasting and testing in cointegrated systems." Journal of Econometrics 35:143-159.

[7] Johansen, S. 1988. "Statistical analysis of cointegration vectors." Journal of Economic Dynamics and Conrrol 12:231-254. 
[8] Stock, J. \& Watson, M. 1988. "Testing for common trends." Journal of the American Statistical Association 83:1097-1109.

[9] Granger, C.W.J. \& Lee, T.W. 1988. "Multicointegration." In Fomby, T.B. \& Rhodes, G.F. eds., Advances in Econometrics; Cointegration, Spurious Regressions and Unit Roots. Greenwich, Ct: JAI Press.

[10] Campbell, J.Y. \& Shiller, R.J. 1987. "Cointegration and tests of present value models." Journal of Political Economy 95:5.

[11] Engle,R.F., Granger, C.W.J. \& Hallman, J.J. 1989. "Merging shortand long-run forecasts. An application of seasonal cointegration to monthly electricity salcs forecasting." Journal of Econometrics 40:45-62.

[12] Engle, R.F. \& Yoo, B.S. 1987. "Cointegrated economic time series: an overview with new results." Paper presented by invitation for the European Meeting of the Economctric Society in Copenhagen.

[13] MacKinnon, J.G. 1990. "Critical values for cointegration tests." Working Paper No. 90-4, University of California at San Diego.

[14] Granger, C.W.J. 1991. "Some recent generalizations of cointegration and the analysis of long-run relationships." Writen for this issue.

\section{Referências}

Dickey, D.A. \& Fuller, W.A. (1979). "Distribution for the estimators for autoregressive time series with a unit root." Journal of the American Statistical Association 74:427-431.

Granger, C.W.J. \& Newbold, P. (1974). "Spurious regressions in econometrics." Journal of Econometrics 26:1045-1066.

Nelson, C.R. \& Plosser, C. (1982). "Trends and random walks in macroeconomic time series." Journal of Monetary Economics 10: $139-162$. 